\title{
Objects of Hypothetical Life on Venus at the Venera Landers Sides
}

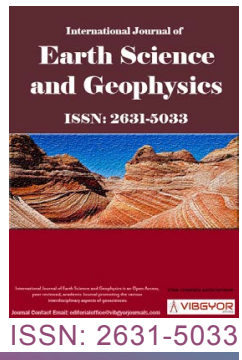

\section{Ksanfomality", AS Selivanov and Yu M Gektin}

Space Research Institute of the Russian Academy of Sciences, Russia

\begin{abstract}
In 1975 and 1982, six TV-experiments on the surface of the planet Venus series were performed by Soviet landers of the VENERA. Recently their images were re-examined using modern processing techniques. As a result of these studies, rather specific objects were observed. In their morphology, some of them recalled Earth living forms. Their striking similarity to terrestrial forms was called terramorphism. The number of detected objects of the supposed forms of the flora and fauna at VENERA landing series reaches 15 . They were found at 3 different landing points, separated by thousands of kilometers. In this paper the authors propose a description of few objects the form and nature of which are discussed.
\end{abstract}

\section{Keywords}

VENERA missions, Extraterrestrial life, Venus fauna and flora, Terramorphism

\section{Introduction}

For thousands of years, humanity has wondered whether there is life outside the Earth Experiments onboard the VENERA landers were designed to gain general notions about the surface of the planet. It did not occur to anybody at that time to search for life on the planet Venus. The ideas of searching for extraterrestrial life usually are based on the postulate of "normal" physical settings, that is, pressure, temperature and, possibly, the composition of the atmosphere of a planet, similar to those present on Earth. But such an approach can be regarded as "terrestrial chauvinism". On the same postulate a term "habitable zone" of exoplanets orbits is based, also taken as an axiom. However, judging by the statistical data on exoplanets, physical settings close to the conditions on the planet Venus are much more often encountered.

We should recall that the Venusian atmosphere almost completely consists of gaseous carbon dioxide, and the cloud layer is formed by micron-sized droplets of concentrated sulfuric acid. The averaged temperature on the Venusian surface is $735 \mathrm{~K}$ $\left(460^{\circ} \mathrm{C}\right)$, and atmospheric pressure attains $9.2 \mathrm{MPa}$. Therefore, Venus itself, with its dense and hot oxygen-free $\mathrm{CO}_{2}$ - high pressure atmosphere can be a natural laboratory for research of this kind. In many regions, the planet surface has the form of either solidified lava or lithified dust deposits. The day illuminance attains $5 \mathrm{klx}$ and higher. The atmosphere absorbs blue rays and the sky is of a yellowish col-

*Corresponding author: LV Ksanfomality, Space Research Institute of the Russian Academy of Sciences, Profsoyuznaya str., 84/32, Moscow, 117997, Russia

Accepted: September 28, 2019; Published: September 30, 2019

Copyright: @ 2019 Ksanfomality LV, et al. This is an open-access article distributed under the terms of the Creative Commons Attribution License, which permits unrestricted use, distribution, and reproduction in any medium, provided the original author and source are credited.

Ksanfomality et al. Int J Earth Sci Geophys 2019, 5:026

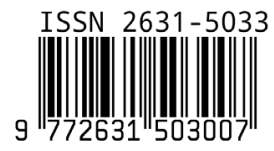


or. The solar disk is hardly distinguished through the permanently present high-altitude cloud layer. The daytime and nighttime durations are 58.4 Earth days each.

The only direct data available on the surface of the planet are the results obtained from television experiments on VENERA landers in 1975 and 1982 [1-3]. In TV experiments on VENERA landers (1975, October, and 1982, March), 41 panoramas (or their fragments) of the surface of Venus were obtained. In the following 43 and 36 years there were no similar missions to Venus; the results of these missions remain unique. They were studied anew, including panoramas that had not previously been analyzed. Several relatively large objects were found, ranging in size from a decimeter to a meter, with an ordered morphology and symmetry reminiscent of the shapes of terrestrial creatures (like small quadrupeds, lizards, snakes and birds). Their striking similarity to terrestrial forms and plants was called terramorphism. Terramorphism intrinsic to certain types of hypothetical Venusian fauna, apparently, relates to the deepest puzzles of searching for life in the Universe. If objects existing under quite different physical conditions exhibit, nevertheless, significant similarity of their shapes, then this phenomenon should be based on certain general natural regularities not yet understood.

As was noted earlier, identification of various objects on the surface of Venus as examples of Venusian flora and fauna is hypothetical, and the (current) names proposed for them, such as "Amisada"

(a)

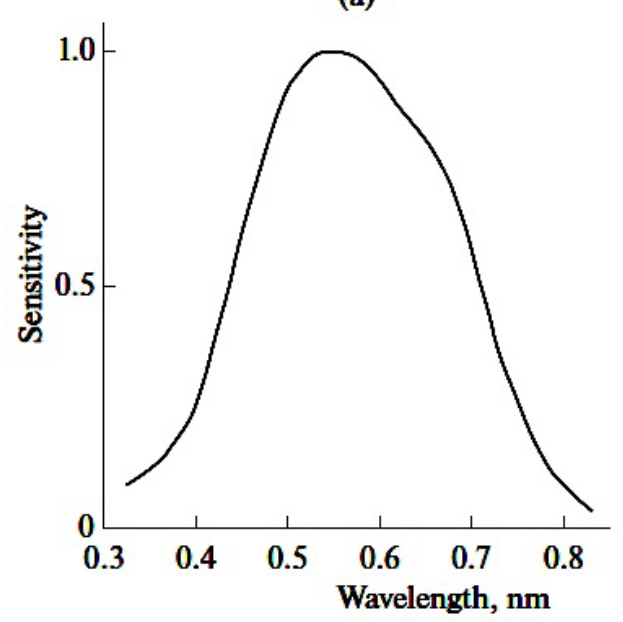

and others, remain conditional.

In the course of observations, some objects changed their shape or moved, very slowly. One of these objects (the "scorpion") is seen only in one panorama, but absent in the others. The number of detected objects of the supposed forms of the flora and fauna of Venus, reaches 15. Few of them are described below.

The results presented below correspond to the missions from which images were returned (VENERA-9, -10, -13, -14). Two mechano-optic cameras were mounted on opposite sides of the lander [4]. The camera's aperture was located at a height of $0.9 \mathrm{~m}$ over the surface, and the upper boundary of the panoramas at the center was at a distance of 2 $\mathrm{m}$ from the spacecraft landing buffer. The spectral intervals (Figure 1) for VENERA -13, -14 cameras were 410 to $750 \mathrm{~nm}$ (no filter), 490 to $610 \mathrm{~nm}$ (a green filter), and 590 to $720 \mathrm{~nm}$ (a red filter). Images of VENERA -9, -10 were black-and-white. The axes of the scanning cameras were tilted at an angle of $50^{\circ}$ from the vertical line, which allowed for resolving millimeter details of the surface in the immediate vicinity of the lander and those of about $10 \mathrm{~m}$ near the horizon (at a distance of $3.3 \mathrm{~km}$ on a flat surface). However, the tilt of the camera axis led to geometric distortions. If one transforms the image in such a way as to correct the line of horizon, the panorama becomes a figure bounded by two arcs, as shown below (Figure 2).

Obtaining a whole panorama with its real-time transfer through the VENERA satellite to the Earth took 13 minutes for VENERA-13, -14 and $30 \mathrm{~min}$ -

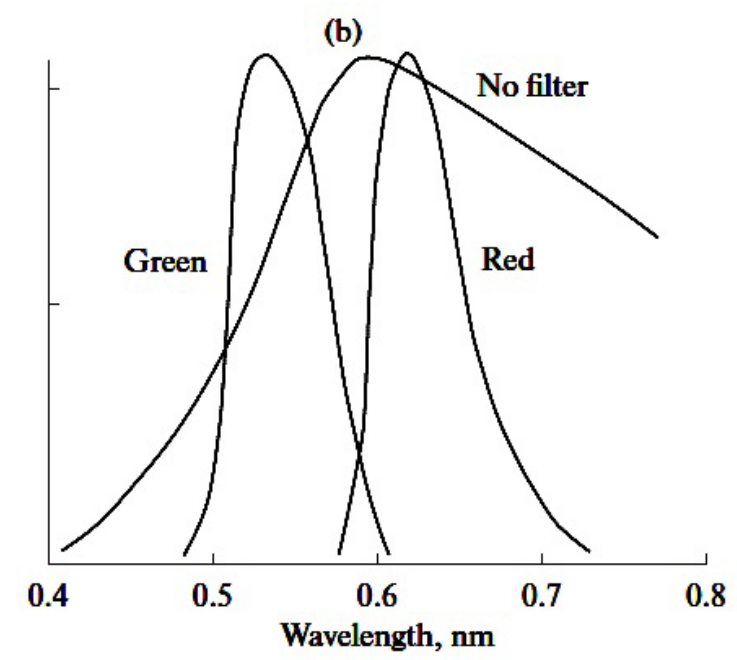

Figure 1: Spectral characteristics of TV-cameras of VENERA-9 and -10 (a) and VENERA-13 and -14 (b) Landers. 


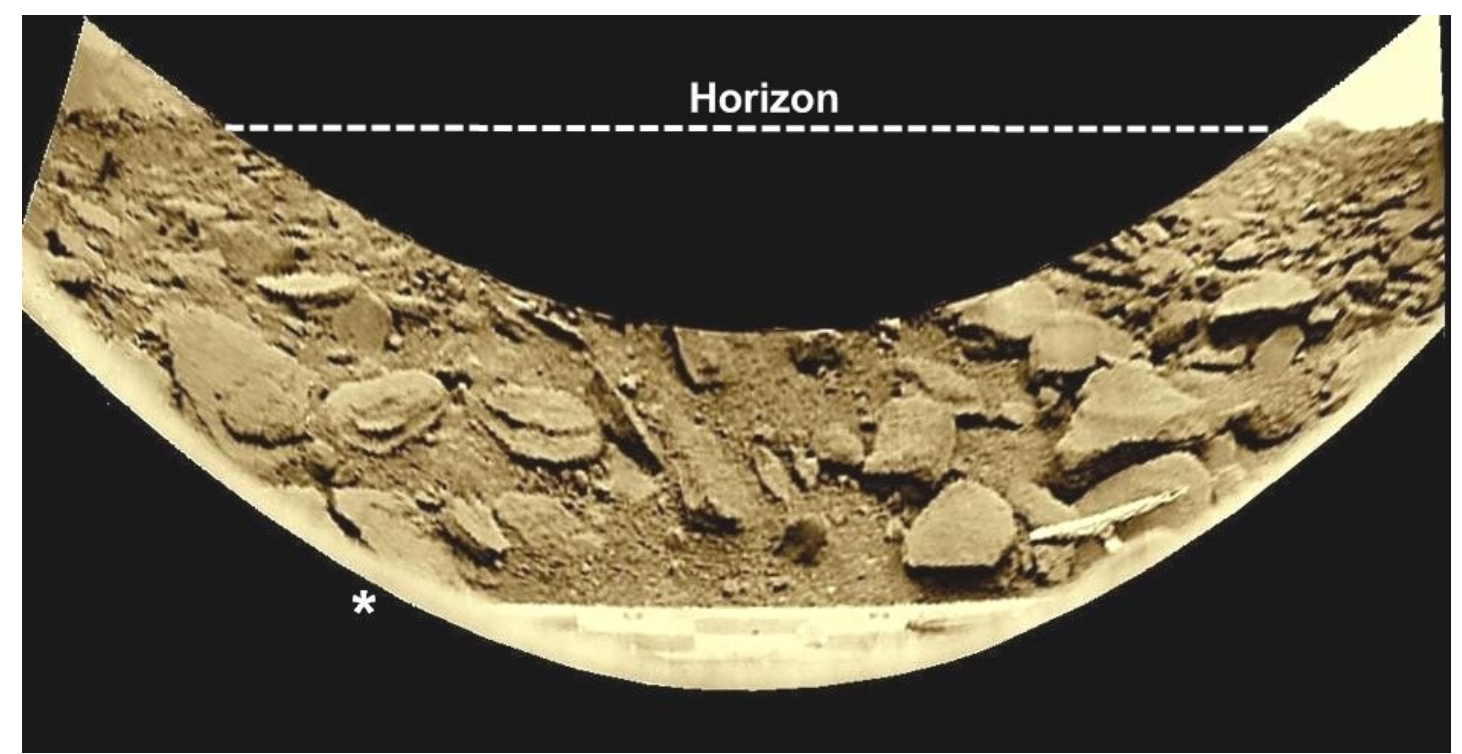

Figure 2: TV-panorama obtained in the VENERA-9 mission, upon additional modern data processing.

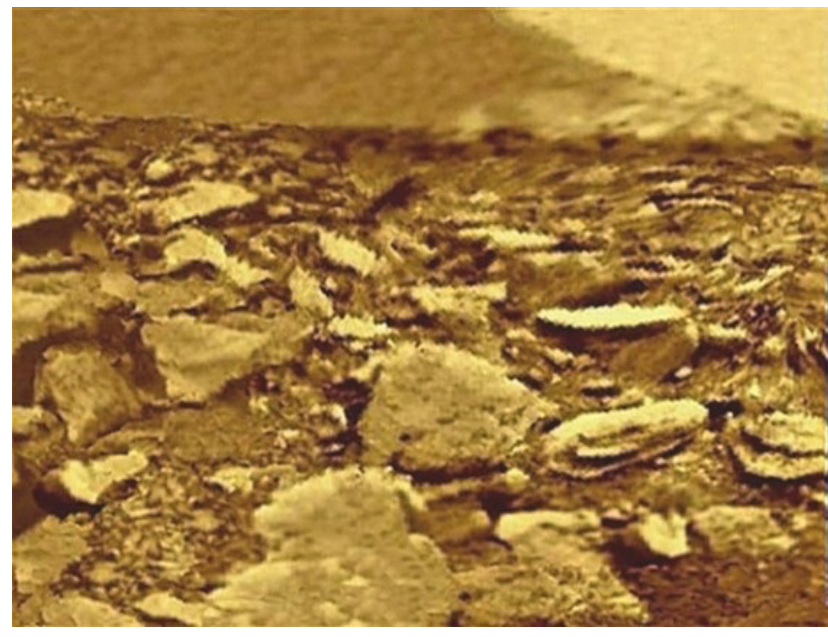

Figure 3: Image of the planet's surface based on VENERA-9 panoramas of the landing site (1975) in modern processing.

utes for VENERA -9, -10. Each image of VENERA-13, -14 cameras consisted of 1000 vertical lines with a resolution of 211 pixels per line, 11 arc minutes each.

With the scan duration of $0.82 \mathrm{~s}$ per line, each pixel was transmitted for $3.3 \mathrm{~ms}$. The duration of the VENERA-13, -14 cameras working time on Venus surface exceeded 2 hours.

On October 22, 1975, the first lander VENERA-9 landed on the planetary surface, at $31.7^{\circ} \mathrm{N}, 290.8$ ${ }^{\circ} \mathrm{W}$, on the day side of the planet Venus. Its cameras transmited black-and-white images [2]. The resolution of cameras of VENERA- 9 was of $21^{\prime}$. The panorama consisted of 517 vertical lines, 115 pixels each. The sweep duration of a single panorama was 30 minutes. The panoramas of VENERA-9 were of low noise but the telemetry data occupied a part of the picture. It was replaced by fragments of the second partial panorama.

The archive data of the television experiment were reprocessed recently, which significantly improved the images resolution and quality (Figure 2). Its left part - a collage image of the landing site with a distant valley - is shown in Figure 3.

Despite the initial low resolution of images, a number of unusual objects were found on VENERA-9 images. Analysis of treated VENERA-9 panoramic images [5] revealed objects that might indicate the presence on the planet of hypothetical forms of Venusian fauna and flora.

The lander's VENERA-13 (March 1, 1982) landing site coordinates were $7.5^{\circ} \mathrm{S}, 303.5^{\circ} \mathrm{E}$, and its height above the level of radius $6051 \mathrm{~km}$ was 1.9 $\mathrm{km}[6]$. The temperature was $735 \mathrm{~K}\left(462{ }^{\circ} \mathrm{C}\right)$ and the pressure was $8.87 \mathrm{MPa}$, which corresponds to the atmospheric density $59.5 \mathrm{~kg} / \mathrm{m}^{3}$, with the composition: $\mathrm{CO}_{2}(96.5 \%)$ and $\mathrm{N}_{2}(3.5 \%)$. The local time was 10:00 am, and the solar zenith was at an angle of $37^{\circ}$.

The view of the planet's surface at the landing site of the VENERA-13 is shown on Figure 4. Illumination by the diffused sunlight was 3-3.5 kLux. Since the rotation axis of the planet is almost normal to the orbital plane, seasonal effects are absent; day and night are equal to each other and last 


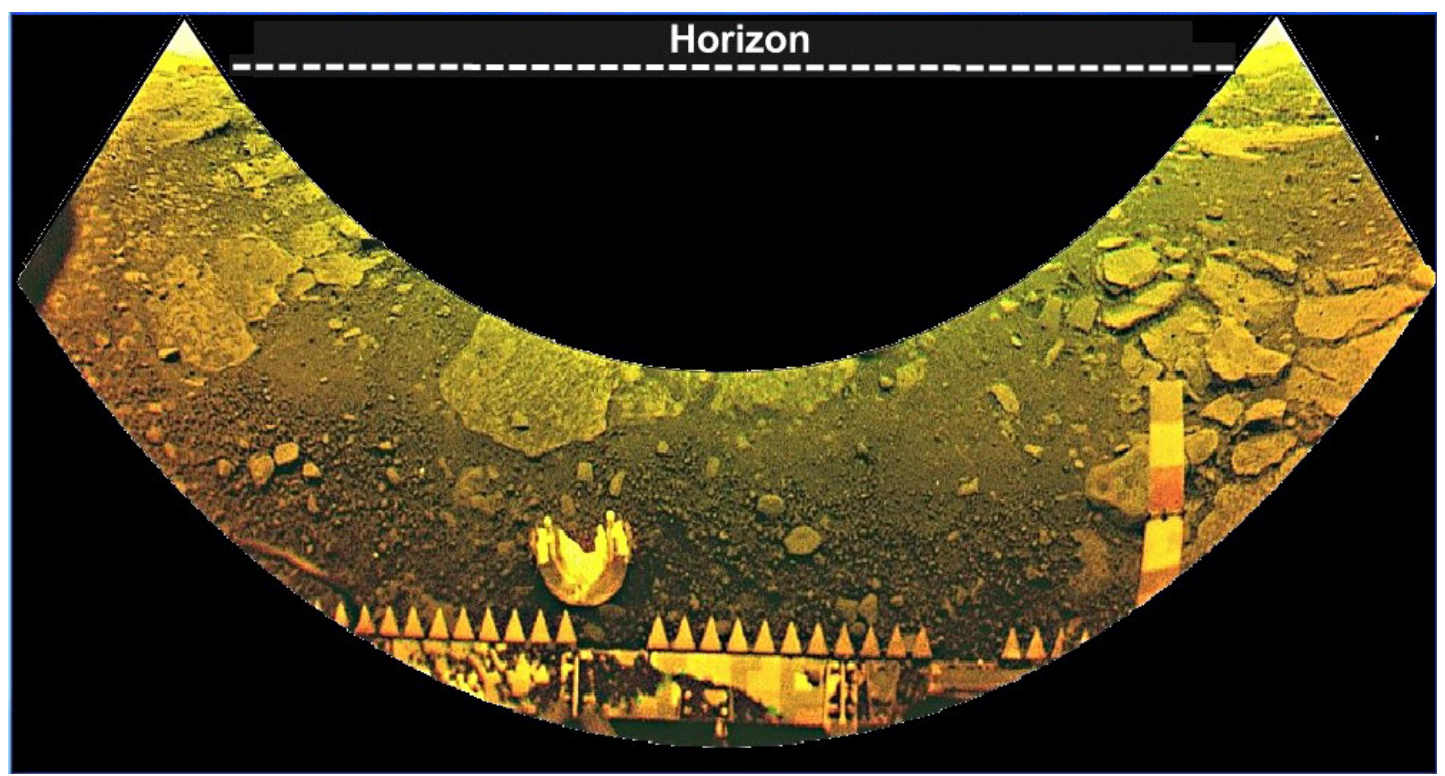

Figure 4: View of the planet's surface at the landing site of the VENERA -13 lander. The surface is crushed soil with stone slabs.

\section{4 days each.}

When experience using image processing was accumulated, the VENERA-13 panorama allowed an approach to the finer details. An important role was played by additional image processing, image geometric correction and the presence of up to eight duplicates of images that were obtained with good quality and low levels of noise. This arrangement enabled the selection and staking of their fragments. As a result, it managed to find and learn about a few new types of hypothetical living creatures at the VENERA-13 and VENERA-14 landing sites.

\section{Some Finds on the VENERA TV Panoramas}

In both data reported above and in previous publications based on TV experiments on the Venusian surface, some objects were recognized whose morphology and behavior resembled terrestrial living forms. All the objects found, basically, were static or extremely sluggish.

Several unusual discovered objects at the VENERA-13 landing cite were presented in [7]. They are related to hypothetical forms of Venusian life. Besides items of hypothetical fauna, there are features found in VENERA images, having a shape of stems and even flowers. Descriptions of them were considered and analyzed in the published papers $[4,8]$. The first stem-like object was detected due to its being close to the entrance of the TV-cam- era, and others were detected by similarities in their shapes and positions to the first "stem". It has a large bulge at the top end, a "burgeon", with a lighter center. There are other stems where the "burgeon" is observed developing to a flower [4]. Here we show one more type of hypothetical venusian life.

"Mushroom" (VENERA-13): One of the findings on the panorama obtained with camera 1 of the VENERA-13 lander relates to one more kind of VENERA finds. This object was located in the foreground, relatively close to the VENERA-13 camera lens at a distance of 15 to $20 \mathrm{~cm}$ from the landing buffer. The object exhibits explicit similarity to terrestrial mushrooms and is supplied with folded cap (Figure 1 and Figure 5). The "mushroom" object has clearly expressed terramorphic attributes, which, again, indicates biophysical regularities yet unknown by science at present.

The "mushroom" is elevated above the surface by about $3 \mathrm{~cm}$; however, its support is not visible. The diameter of the "mushroom" attains, approximately, 8-9 cm. It is clearly seen on all sequential panoramas of VENERA-13. Despite the object resides in the shadow region under the parachute panel of the lander, the mushroom is the brightest object in the central part of the panorama. The color image of the area is composed of black-andwhite and color-divided red and green primary panoramas. Six sequential images were processed by the method of correlative stacking, including 


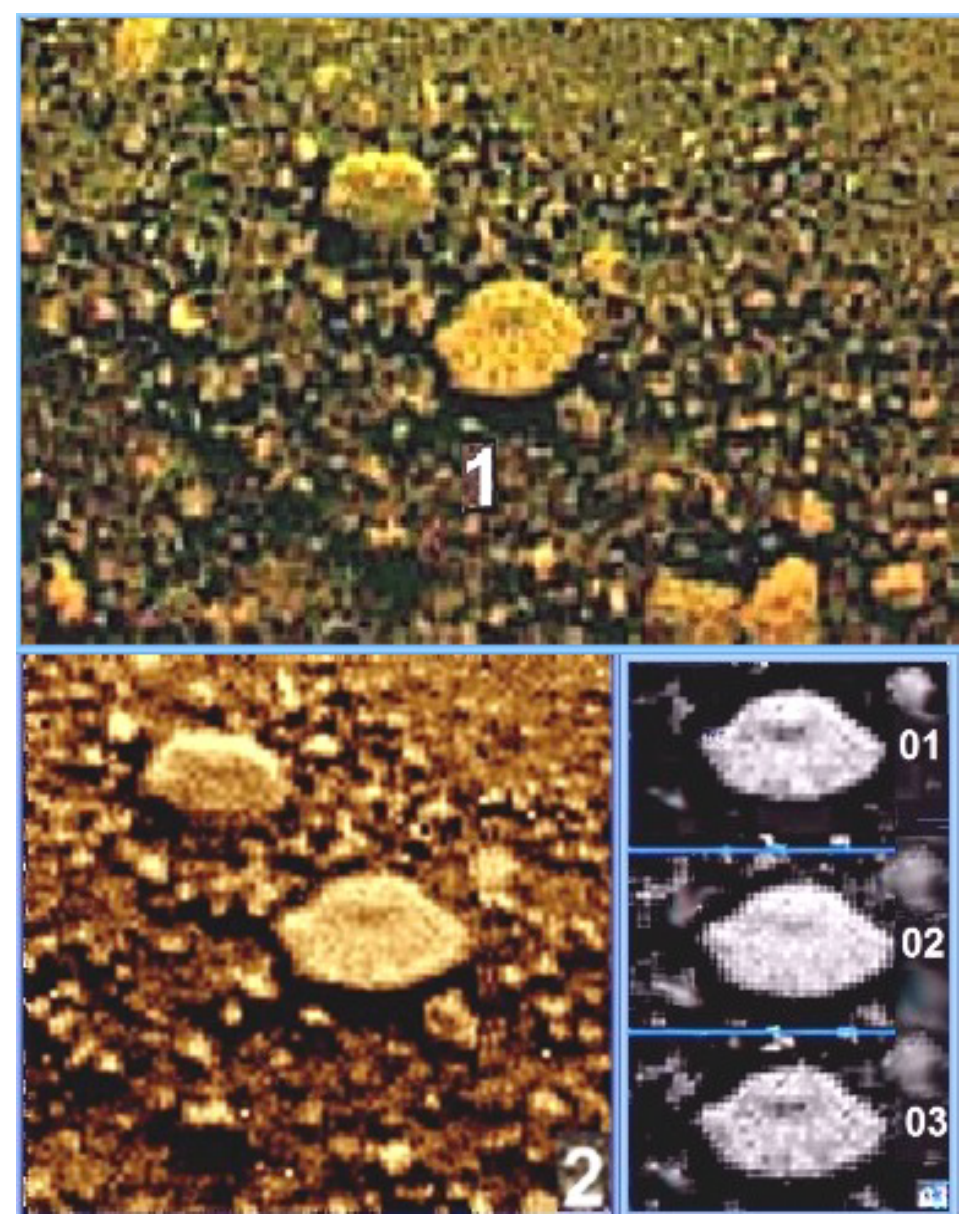

Figure 5: (1) Central part of the VENERA-13 panorama with the object reminiscent a mushroom (object size is close to $8 \mathrm{~cm}$, and it is elevated above the surface, by approximately $3 \mathrm{~cm}$ ). (2) Fragment of the panorama after new processing (with the corrected geometry) and three different versions of the results after the combined processing of fragments of six panoramas. The radial folded structure of the object surface is similar to that of many kinds of terrestrial mushrooms.

panoramas that had not been used previously. Three of the resulting versions of black-and-white images processed are shown in right hand side of Figure 2 and Figure 5. In each case, the conically folded tent-shaped structure of the object is seen. There are two black points at the cone's top. For the 2-hour long mission on the Venusian surface, no attributes that could testify to motion of the object were found. Apparently, one may relate this object to hypothetical Venusian mushrooms.

The radially-folded form of the surface of the "mushroom cap" repeats their classical form, as of many mushrooms of the Earth. However, the "classical" form does not exhaust the findings on the panorama of VENERA-13. On the right, at the color test panel, there is another object of regular shape (Figure 6), about $8 \mathrm{~cm}$ in size, very similar to the terrestrial tree fungus, in the form of an edged

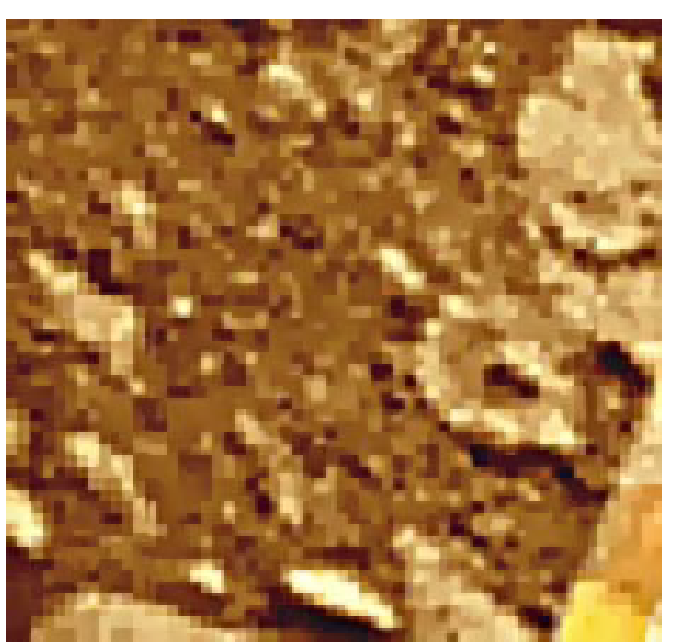

Figure 6: Another object of hypothetical Venusian biosphere was located relatively close to the VENERA-13 color test panel. The object's shape exhibits explicit similarity to terrestrial tree fungus. 


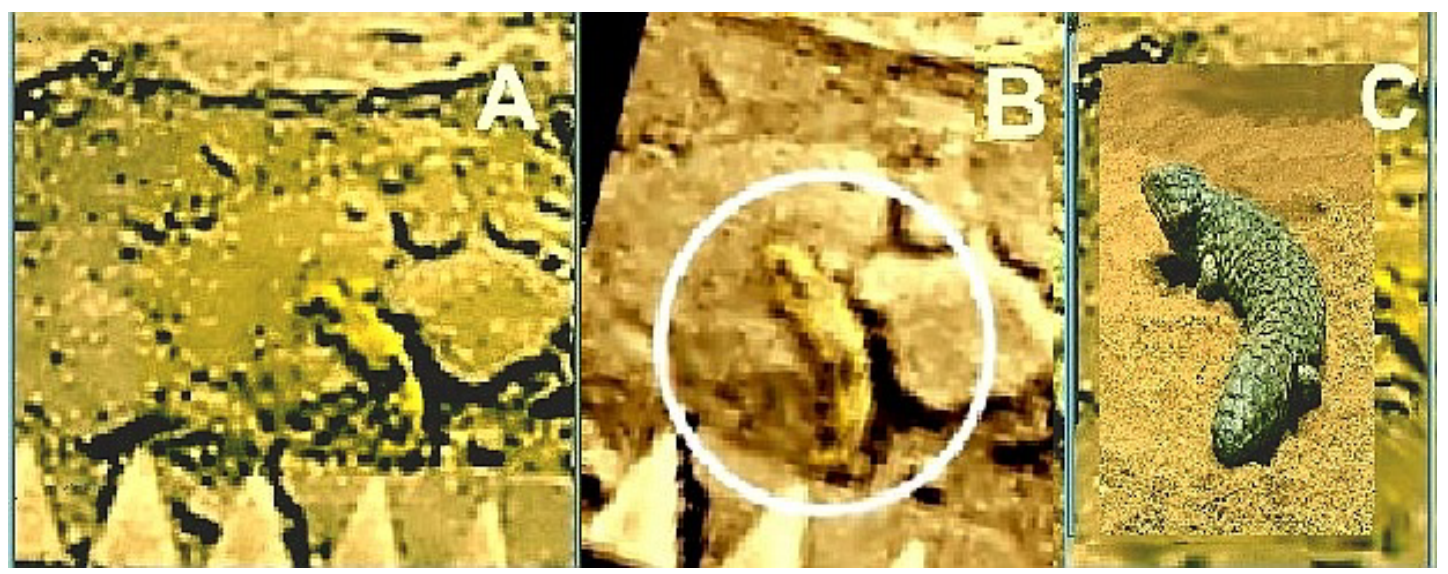

Figure 7: (A) Amisada climbing up a stone, (B) stacking of 6 original fragments of VENERA-14 panoramas, (C) sluggish Australian Shingle back lizard whose size and shape resemble the amisada.

half-disk. On Earth, such tree fungi are often found in forests of middle latitudes as growths on tree trunks.

By virtue of the fortunate close position of the "mushrooms", their structural details are clearly distinguishable. Again, this allows associating their properties with the most noticeable manifestations of terramorphism. According to the principles of biological classification, the "kingdom of fungi" is independent and does not enter into the fauna or flora kingdoms.

Amisada (VENERA-14): The "amisada" was found in the central part of the VENERA-14 panorama. It is located very close to probe landing buffer. Amisada is highlighted by a white circle in Figure 7. The recognized hypothetic living object related to the hesperas class is the amisada resting close to the input window of the VENERA-14 TV camera. The conditional term "amisada" originates from the shortened name "Ammizaduqa" of the Babylonian king (sixteenth century BC). At that time, ancient astronomers started to register on ceramic tables the time of morning and evening elongations of Venus.

The shape of the amisada resembles a lizard climbing up a stone. The spotty amisada were located in the immediate vicinity of the landing buffer, close to the TV-camera lens; therefore, we managed to resolve some of its fine details. By virtue of the low noise TV images of VENERA-13 and -14 , it is possible to see and to recognize fine and slow displacements of the amisada's forward (upper) parts (if actually it is its forward part). In Figure 7A, a single image of the amisada is presented, which cor- responds to about the $30^{\text {th }}$ minute of activity of the TV camera of the VENERA-14 lander. In Figure 7B, the averaged shape (for 6 original images) of the amisada is shown. Further, in Figure $7 C$, the sluggish Australian Shingleback lizard is also presented, which according to its size $(10-15 \mathrm{~cm})$ and tardiness resembles the amisada.

The amisada is seen from above at an angle of about $80^{\circ}$ to the horizon. In contrast to the other figures, we see that the amisada is in a slow motion. Amisada, apparently, is climbing up or creeping onto a $5-8 \mathrm{~cm}$ stone. The upper part of amisada is already residing on the flat surface of the stone, whereas the lower part envelopes the ledge. Of course, we cannot exclude the possibility that amisada is not rising but going down the stone. Probably, the two small outstanding details on the head part of the amisada are its forelimbs. As a whole, amisada resembles a lizard, which again indicates surprising terramorphic features of Venusian fauna. In Figure 7A and Figure 7B, spots on the body of amisada are distinguished. An elongated spot is seen on the neck part of amisada, whereas enveloping dark spots are visible in both the central and the lower body parts. The length of the amisada is about $10-12 \mathrm{~cm}$. There is another more distant amisada nearby.

The experimental method employed did not allow the dynamic of the objects to be observed, insofar as each panorama was swept and simultaneously transmitted through the radio line for a rather long time interval. However, we were able to compare sequentially the panoramas obtained as instantaneous photographic pictures. In a number 


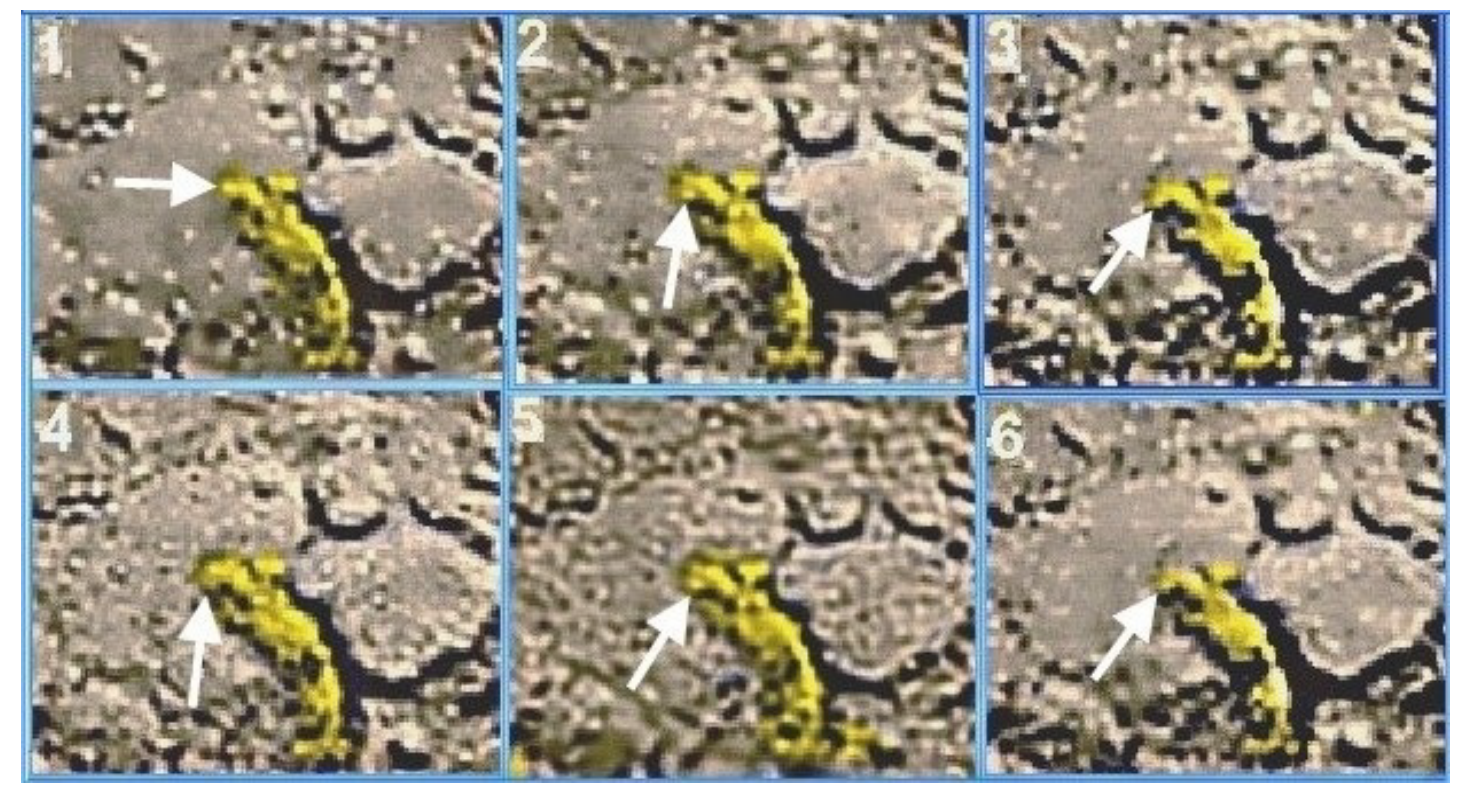

Figure 8: Non-averaged processed fragments of original panoramas. Positions of arrows indicate the sequentially varied directions of the forward part of the amisada, whose size and motion resemble a human finger. Arrows indicate how the forward part changes its direction. All original Images are black-and-white.

of cases, this made it possible to observe the distinguished displacements of objects or their escape outside of the limits of the field of observation.

Some of them recall explicit traces of their displacement. This also allowed us to suppose their ability to move and even to find velocities available for them. For amisadas, the displacement velocities

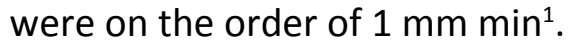

Images of the amisada were additionally processed, which made it possible to demonstrate the motion of its forward part as a sequence of its position. Variation of the forward part of the amisada position is shown in Figure 8 as changes in the direction of arrows. Here, fragments of the six available panoramas are given. The images are presented in chronological order, with the intervals between them being, on average, of about $13 \mathrm{~min}$. Over 1.5 hours, the head part of amisada gradually shifted. In frame 1 of Figure 8, the upper part fragment of the amisada is directed to the left (the 9h), whereas the shadow under it is almost not visible. We should recall that for scattered natural illumination on the Venusian surface, shadows appear only in the case of low positions of an object with respect to the surface. In this case, the altitude of the object above the surface should be comparable to its size. Most likely, the absence of a shadow indicates that the object is lifted above the surface. In frame 2, the amisada's forward fragment is dis- placed along the 7-h direction, and no shadow is also present. Finally, in frame 3 , the edge fragment corresponds to, approximately, the 8-h direction with a dense shadow under the object. Further variations are given by the frame sequence 4 to 6. The size and displacements of the fragment are close to those of a human finger and its motions. The displacement, in itself, attains 1 to $2 \mathrm{~cm}$. This value seems may be housekeeping action. Is the amisada looking for food?

The camera lens resolution is insufficient for the detection of fine details, such as grass or moss. If the hypothetical Venusian fauna is heterotrophic, the source of its existence should be hypothetical autotrophic flora. Direct rays of the Sun, as a rule, do not reach the surface of the planet, nevertheless there is enough light for photosynthesis of the Earth-like type there. Of course, photosynthesis at high temperatures and in a non-oxidizing environment should be based on a completely different, unknown biophysical mechanism.

Together with items of terramorphic shape, there are objects the form and nature of which remains, at least partly, incomprehensible. In this paper we propose one of them nicknamed "Jaggy".

"Jaggy" (VENERA-9): On processed VENERA-9 panorama considered in [9], a large elongated bright object stands in having the size about of 1.3 $\mathrm{m}$, with a strange jagged edges, by its contours re- 


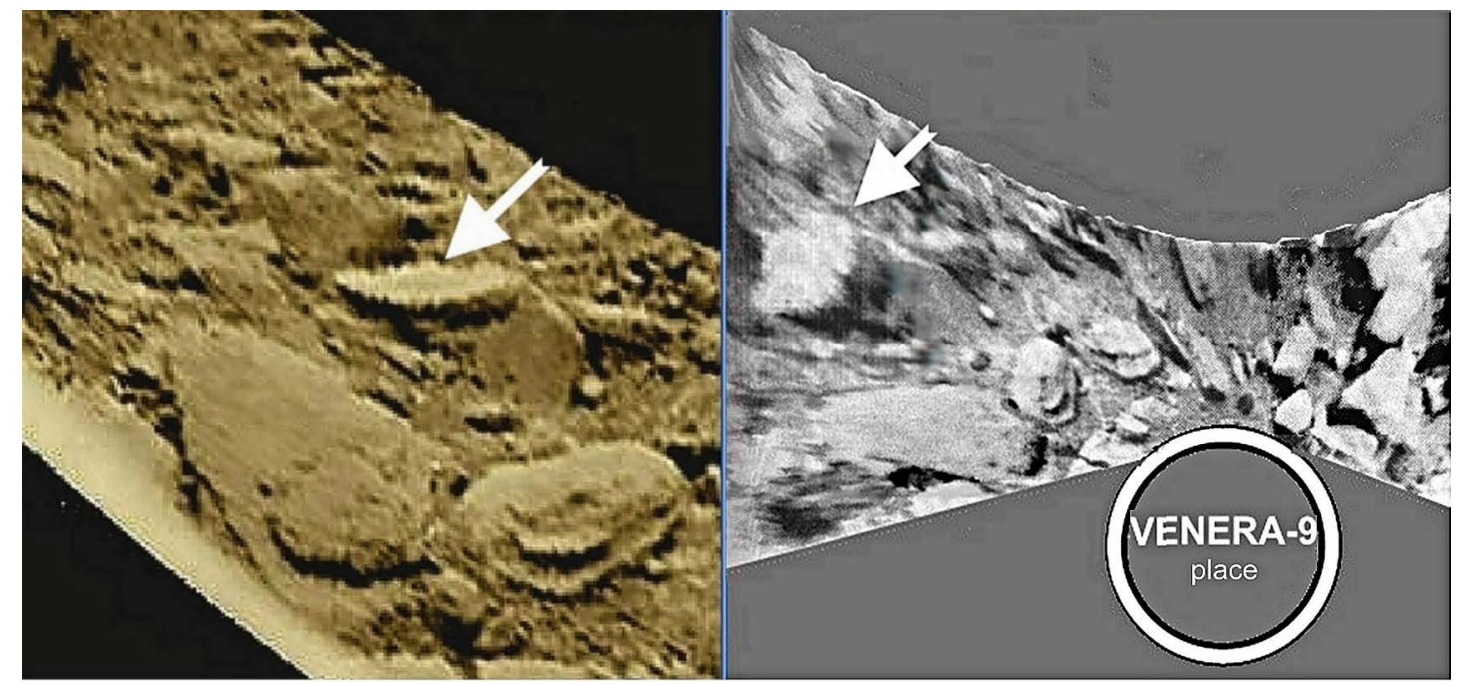

Figure 9: "Jaggy" object (arrow) on the processed VENERA-9 panorama (left) and its horizontal projection (photo plan, right). The circle shows the position and size $(2 \mathrm{~m})$ of the VENERA-9 landing buffer.

sembling earthly jagged leafs of trees (Figure 9). Initially it was supposed that its jagged edges are results of a linear structure of the TV image, however it is not a case for surrounding details. It is nicknamed "Jaggy" and is much lighter than the surface of surrounding stones. "Jaggy" rose above the surface, with a deep shadow visible under it. "Jaggy" is large, however due to the large distance from the camera (approximately $4 \mathrm{~m}$ ), the object structure is difficult to define. The photo plan could help. Unfortunately, there is only a working version of the photo plan, based on the VENERA-9 primary data processing. Nevertheless, it can be seen that in comparison with the surrounding boulders, the "Jaggy's" structure is more complicated; guessed is a radially and radiant appearance of its periphery (Figure 9, right side). The photo plan one may considered as a horizontal projection of "Jaggy" (which by its shape is more reminiscent of a sea stingray). The photo plan resolution is low and does not allow drawing any specific conclusions about the nature of "Jaggy" and, even more, to attribute it to the flora or fauna. Jaggy rests on a stone. The elongated shape of details in the edge of the object (Figure 9, right side) may remind feathers. They are the more pronounced, the clother they are to the edge of Jaggy. There is a shadow under Jaggy repeating its shape. It should be recalled again that the resolution of the VENERA-9 camera was 21', twice worse than that of the VENERA-13 and -14 cameras; so attempts of identifying the "Jaggy" details should be treated with caution.

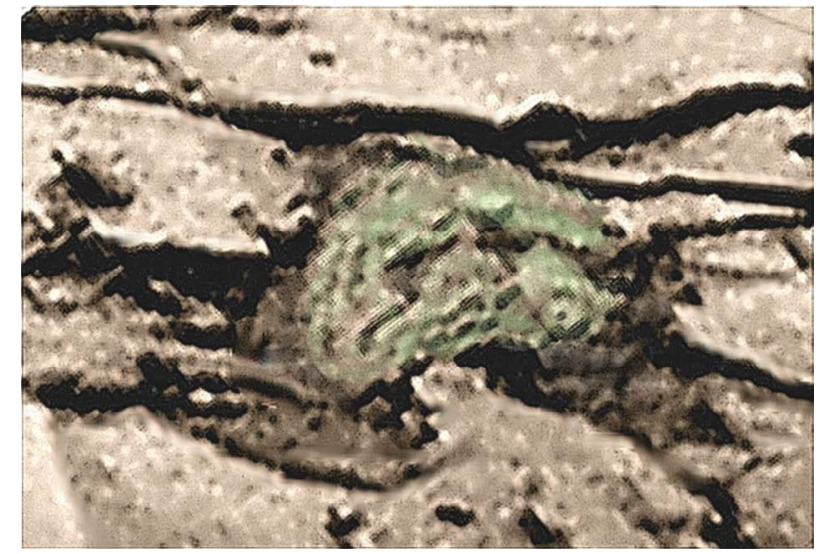

Figure 10: Fragment of the VENERA-14 panorama. A coiled snake-shaped object is seen in a small depression. Its greenish color is exaggerated.

Nevertheless, the symmetrical form of Jaggy on the photo plan allows us to suspect in it an object resembling a bird with almost 1.5-meter span of wings and protruding a $10-15 \mathrm{~cm}$ "tail". So, isn't it a bird of Venus that we see in Figure 9? It should been noted that the dense atmosphere of Venus would be a convenient environment for the flight of hypothetical living beings.

Hunting on Venus (VENERA-14): The resolution of the VENERA cameras, limited by an angle of 11 ', did not allow detailed studies of objects outside the 4-5 m. But if the properties of nearby objects could be established, the more distant ones can be detected by the similarity of their shape. So we succeeded to find the second "snake", which, apparently, was engaged in hunting. The slowness 


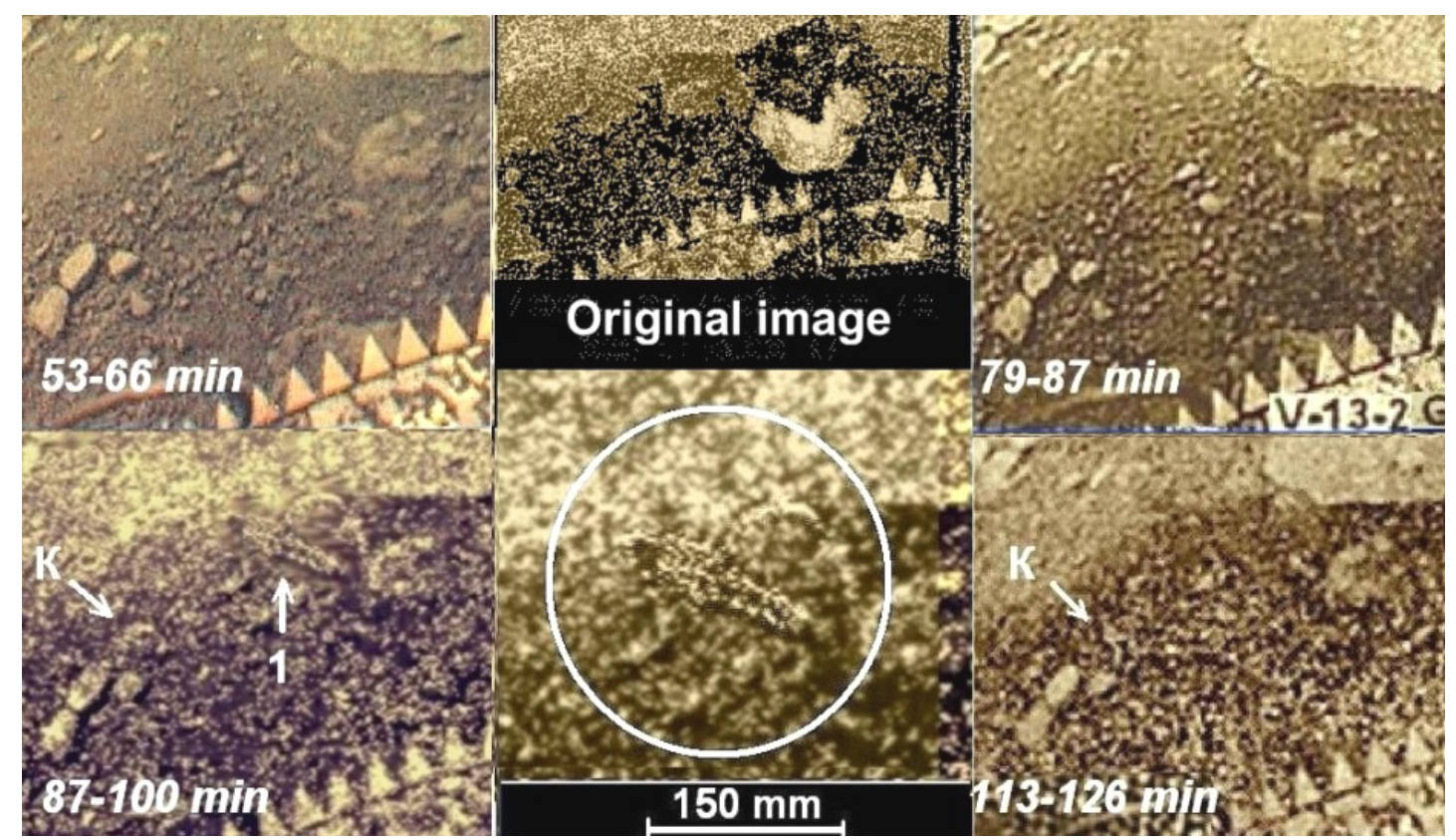

Figure 11: The object "scorpion" appeared in the panorama 6BW obtained from the $87^{\text {th }}$ to the $100^{\text {th }}$ minutes. In images obtained before the $87^{\text {th }}$ and after the $113^{\text {th }} \mathrm{min}$, the object is absent. (In the images of minutes 87 100 and 113-126 min, to the left, in the group of stones, there emerged a new object $K$ with a changing shape). The central part of the figure, at the bottom, shows the result of the "scorpion" image processing and its size.

of living forms on Venus has already been noted above. The snake curled into a ring (Figure 10) was described in [9].

The animation, composed of the successive panoramas of VENERA-14, shows that the vital processes under Venusian conditions take place at a different pace than on Earth. It is most likely that the second snake on the VENERA-14 panoramas was found in the process of one of amisadas hunt. As follows from the animation made up of the successive panoramas, the hunting process developed very slowly. Unlike the half-meter eye-catcher "snake", the hunter with a large head straightened into a body longer than a meter. Snake, apparently, noticed the wildfowl and began to hunt. As can be seen from the successive panoramas, at first her head with a large eye is visible in profile. It crawled to the amisade, according to its concepts, quickly - overcame 2 meters just a couple of Earth' hours. The snake was already $15 \mathrm{~cm}$ from the victim and stopped, turning its head slightly to assess the hunting prospects. This took another 26 minutes. In this image, both eyes are visible, and its body bent. The wildfowl was an amisade. Amisada, finally, realized the danger and recoiled, for whole $4 \mathrm{~cm}$. It took 13 minutes. Unfortunately, what happened next is unknown; After working for 2 hours at a temperature of $462^{\circ} \mathrm{C}$, the VENERA-14 lander began to be turned off, and its television cameras were off. The event took place on the planet Venus, on March 5, 1982 at 10 am local time. The time itself on Venus is unhurried: day and night last here for 58.5 terrestrial days, and the year of Venus consists of two (more precisely, 1.91) solar days.

Scorpion (VENERA-13): The first detected object, conditionally called "the scorpion", was discovered in the panorama $6 \mathrm{BW}$, near the $90^{\text {th }} \mathrm{min}$ ute of scanning [7]. Before that, the instrument had already been working for 1 hour and 27 minutes (moment of the start of scanning the 6BW image); therefore, our first suggestion was that this regular structure is a product of destruction of some part of the lander itself. But the lander VENERA-13 continued to work for almost a whole hour after that, indicating that there could not be any destruction, otherwise all instruments would have failed due to catastrophic overheating immediately. The assumption of a split detail is also in conflict with the fact that the object is absent in the subsequent images.

The history of the emergence and disappearance of the "scorpion" is illustrated in Figure 
11. In images obtained before the $87^{\text {th }}$ minute, the object is absent. The object is absent as well in the later image 6G (113 to $126 \mathrm{~min}$ ). A likely cause may be in that, if the object moved, then, as it went away to a distance of 3 to 4 meters, it became indistinguishable from stones. At such a distance, as a minimum, the object should have moved away in 26 minutes, the time up to the next panorama 6G in Figure 11.

In the course of scanning, the image of a moving object can be distorted. The image fragment with the "scorpion" was obtained during 32 seconds. The object motion could cause, for instance, its seeming lengthening or shortening in the image. However, later it was found that all hypothetical living objects that show movement, are very sluggish. Their speed doesn't exceed $1 \mathrm{~mm} / \mathrm{s}$.

The "scorpion" has a length of 15 to $17 \mathrm{~cm}$ and a complicated structure resembling some terrestrial arachnida or insects. In its immediate vicinity in the panorama $6 \mathrm{BW}$, there emerged a formation like a half ring of the same size. It is a moving object of another class, which does not disappear but takes another position in each image. In our first paper on "scorpion" we mentioned that the complex and regular shape of the "scorpion" cannot be a result of a random combination of light, half-tone, and dark points. The probability of forming such an image, if we count only the number of combinations, is vanishingly small and is in fact excluded. In addition, there is a physical indication of "scorpion" reality: an analysis reveals a shadow under the object. A shadow shows that the object has a relief and is located over the surface.

A possible explanation of the emergence and then disappearance of the "scorpion" is connected with destruction and lateral ejection of soil in the course of landing rather than a direct influence of the wind [7]. The vertical speed of the spacecraft at landing, found by a dynamical method [6], was $7.6 \mathrm{~m} / \mathrm{s}$, while the lateral speed was approximately the same as that of the wind $(0.3$ to $0.5 \mathrm{~m} / \mathrm{s})$. As a cause of the object emergence and subsequent disappearance, the possible role of the wind was also considered. At the measured wind velocity of $0.48 \mathrm{~m} / \mathrm{s}$ at the landing point [8] and the atmosphere density of 60 $\mathrm{kg} / \mathrm{m}^{3}$, the wind "head" pressure $\rho v^{2} / 2$ on the "scorpion" cross section gives a pressure force of about $0.08 \mathrm{~N}$, which is insufficient for its dis- placement.

Here is another possible explanation. The stroke amounted to $50 \mathrm{~g}$ of Venus. The lander destroyed the soil to a depth of about $5 \mathrm{~cm}$, threw it aside, and the soil could have covered the "scorpion". The place where the "scorpion" appeared was studied in the whole sequence of panoramas, from the $7^{\text {th }}$ to the $119^{\text {th }}$ minute. At first, a shallow ditch of about $100 \mathrm{~mm}$ long is seen on the soil thrown out. Then the sides of the gutter are lifted, and its length grows to about 150 $\mathrm{mm}$. The gutter orientation is the same as that of the "scorpion." In an hour, the regular structure of the "scorpion" emerged from the ditch. At the $93^{\text {rd }}$ minute, the "scorpion" probably completely got out of the soil that had covered it, that was not thicker than 1 to $2 \mathrm{~cm}$. At the $119^{\text {th }}$ minute, it had already gone. Thus, the object needed about an hour and a half for the "rescue" operation. This apparently points to its restricted physical abilities.

The coincidence of the scanning time of the panorama 6BW with the emergence of the "scorpion" object was a great fortune of the experiment. Lucky was also the very position of the survey where the resolution made it possible to follow both the development of the events described and the disappearance of the object in the last panorama.

\section{Conclusion}

The scanning cameras of the VENERA landers (1975 and 1982) were intended to produce a general notion about the planet's surface and did not anticipate finding any possible inhabitants of Venus. Nevertheless, certain unusual findings that have a structure similar to the Earth' fauna and flora were found in different areas of the planet. Besydes, there are objects the form and nature of which remains at least partly incomprehensible. The planet's Venus surface needs for new investigations with better resolution and longer observation. The special mission, if it ever takes place, should be significantly more complex than VENERA probes.

Terrestrial life is based on the water medium. The typical Venusian landscape is a waterless hot stone or friable desert, sometimes, mountains and, rarely, volcanoes. High in the sky, at an altitude of $49 \mathrm{~km}$, resides the lower boundary of the 
clouds consisting of an extended layer of concentrated sulfuric-acid clouds. For temperatures of $460{ }^{\circ} \mathrm{C}$, at the landing sites of the descending landers, water cannot exist in its liquid form. The fraction of water in the gaseous state also is negligible (about of $2 \cdot 10^{-5}$ ). Water, as well as oxygen, are virtually absent on Venus. Thus, the question arises: what material could be used by nature for life on this planet? To answer the question, we have as yet only assumptions. It is well known that the rate of chemical reactions under these temperatures is extremely high. Temperatures close to $732 \mathrm{~K}$ at the planet's surface are unacceptable for terrestrial forms of life. However, from the thermodynamic standpoint, these conditions are not worse than those existing on the Earth. The media providing life on Venus and the chemical agents acting in it are not known, but nobody has searched for them. The original materials on the planet have minor differences from terrestrial ones. At the same time, anaerobic mechanisms are well known in biology. For a number of procariotesy, photosynthesis is based on the chemical reaction in which sulfuric hydrogen $\mathrm{H}_{2} \mathrm{~S}$ but not water turns out to be an electron donor. A number of underground autotrophic prokaryotes use chemosynthesis instead of photosynthesis.

Why was the velocity of all objects related to presumed Venusian fauna for which we have managed to observe the displacement surprisingly low, namely, lower than $1 \mathrm{~mm} \mathrm{~s}^{-1}$ ? The medium constituting the hypothetical living forms on Venus should possess certain unusual properties. One of the assumptions is that this feature is associated with certain energy constraints intrinsic to Venusian fauna. Simultaneously, another, even more likely, reason may exist, which is determined by the properties of the medium constitutng bodies of Venusian fauna. If the existence of liquid water on the Venusian surface is excluded, then living forms there should employ other media to form their structures. For example, these media might be epoxies that exhibit the slowed-down plasticity. A body designed on the basis of this viscous medium requires noticeable time to propagate chemical information inside it and to transform the body's shape. We can assume that the properties of the viscous medium are the reason for the sluggish behavior of the Venusian fauna.
It is assumed that the results presented can be associated with the existence of the Venusian fauna, although there is a large number of questions (energy sources, e.g.), which are not considered here, and the answer to many of them will be not be known soon, perhaps, until after the next mission to Venus. For the present, all hypothetical findings of fauna and flora on Venus require confirmation by subsequent experiments. This story develops slowly and has continued already for 6 years. But, even in the $19^{\text {th }}$ century, the philosopher Arthur Schopenhauer (1788-1860) wrote about new discoveries in science: "Every truth passes through three stages, at the first stage you will be ridiculed, at the second they will fight you violently, at the third your statement will be accepted as being self-evident". We would like to live to stage 3 of our story.

\section{References}

1. Selivanov AS, Chemodanov VP, Naraeva MK (1976) TV Experiment on the Venus Surface. Kosmich Issled 14: 674-677.

2. Keldysh MV (1979) First Panoramas of the Surface of Venus. (In Russian). Moscow, Fizmatlit, 132.

3. Selivanov AS, Gektin Yu M, Gerasimov MA, Nosov BI, Naraeva MK (1983) Continuation of the TV Investigation of Venus surface by means of landers. Kosmich Issled 21: 176-182.

4. Ksanfomality LV, Selivanov AS, Gektin Yu M (2014) Possible signs of flora on the planet venus. Global Journal of Science Frontier Research 14.

5. Ksanfomality LV (2012) Mobility and other features of hypothetical venusian fauna. Doklady Physics 57: 497-501.

6. Baklunov AM, Karyagin VP, Kovtunenko VM (1983) Automatic Interplanetary Stations VENERA-13, -14. Kosmich Issled 21: 151-153.

7. LV Ksanfomality (2013) Possible signs of life on the planet venus. International Journal of Astronomy and Astrophysics (IJAA) 3: 57-79.

8. Ksanfomality LV (2014) Hypothetical flora and fauna of Venus. Acta Astronautica 105: 521-533.

9. Ksanfomality LV (2014) "SNAKE": One more terramorphic entity of the hypothetical Venus fauna. International Letters of Chemistry, Physics and Astronomy (ILSPA) 3: 64-75.

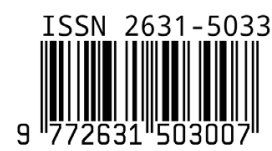

